\title{
Análisis del proceso de especialización regional en servicios en España
}

\author{
Juan R. Cuadrado-Roura. Universidad de Alcalá, Madrid, España. \\ Andrés Maroto-Sánchez. Universidad de Alcalá, Madrid, España.
}

RESUMEN | ¿Ha tenido la especialización productiva en servicios un reflejo uniforme a escala regional? ¿Se ha reducido la desigualdad productiva de las regiones y qué papel desempeñaron los servicios? ¿Cuál ha sido la contribución de los servicios al crecimiento económico de las regiones? Para responder a estas preguntas, tomando el caso de España como referencia, se especifican dos hipótesis. La primera es que el período analizado parecería mostrar una notable inercia en la especialización, lo que determina una baja convergencia de las estructuras productivas. La segunda sugiere que la especialización productiva influye en el crecimiento económico regional y que los servicios han tenido una importante función en este sentido. El análisis utiliza diversos indicadores y técnicas y los resultados confirman en gran medida las hipótesis de partida, aportando detalles y aspectos anteriormente no bien conocidos.

PALABRAS ClAVE | desarrollo regional y local, distribución espacial, desigualdades regionales.

\begin{abstract}
The article deals with three questions. First, if productive specialization on service industries has shown a uniform pattern at regional level. Second, to what extent productive inequalities amongst regions have been reduced or not and which was the role of services. Finally, what has been the contribution of services to regional economic growth. Taking Spain as the casestudy, two hypotheses have been specified to answer these questions. The first is that figures should show an inertial trend of the productive specialization, which has produced a low convergence of regional productive structures. The second suggests that productive specialization has influenced regional economic growth and that services have played an important role in it. Several techniques are used to carry out the analysis. Results confirm to a great extent the hypothesis and make possible to set up some aspects not very well known previously.
\end{abstract}

KEY WORDS | regional and local development, spatial distribution, regional inequalities. 


\section{Introducción}

El análisis de la evolución económica de las regiones, al igual que en el caso de cualquier economía nacional, puede realizarse adoptando diversos enfoques. Dos de ellos destacan particularmente. El primero se centra en intentar comprender y valorar los factores que se consideran básicos para explicar los logros de una economía. El segundo dirige la atención al comportamiento específico de los distintos sectores productivos y el papel que desempeñan en el crecimiento económico. En el primer caso, no solo se tienen en cuenta los factores más convencionales - acumulación de capital, trabajo, tecnología-, sino otros componentes (ahorro interno y externo, población y estructura por edades, ratios de empleo y de participación, productividad, incorporación de nuevas tecnologías...). Cuando el análisis se refiere a economías regionales, hay que tener en cuenta, además, los factores nacionales que tienen influencia directa o indirecta en ellas y aquellos otros que pueden afectarlas o condicionarlas especialmente (evolución cíclica, recursos disponibles en la zona, orientación de las políticas económicas, etcétera). ${ }^{1}$ Evidentemente, el segundo de los enfoques citados no excluye un tratamiento paralelo de los factores básicos de crecimiento, es decir, los que se han citado para el primer enfoque, pero pone su énfasis en la estructura productiva de las economías y en el papel que pueden desempeñar, en cada caso, el sector agrario, la minería y energía, las manufacturas y los servicios. A partir de este segundo planteamiento se han sugerido con frecuencia algunas acciones sectoriales prioritarias orientadas a impulsar el desarrollo de la economía objeto de estudio. Una de las más tradicionales es la que consideró que el desarrollo industrial debía constituir la base sobre la que asentar el crecimiento económico de un país o de una región (Kuznetz, 1974; Prebisch, 1950; Todaro, 1982; Temple, 1994; Thirlwall, 1999; y otros).

Los estudios que relacionan el cambio estructural de las economías con los procesos de crecimiento se vinculan claramente al segundo de los enfoques citados. El tema cuenta con una amplia literatura, generalmente con referencias explícitas a los procesos de reducción de las disparidades económicas y la convergencia entre países y/o regiones (Camagni \& Cappellin, 1985; Raymond, 1990; Suárez-Villa \& Cuadrado-Roura, 1996; Adams \& Pigliaru, 1999; Paci \& Pigliaru, 1999; Garrido, 2002; Cuervo, 2004; Eckey \& Türk, 2007). La conclusión que se deduce de la mayoría de los trabajos que se refieren al comportamiento de las regiones de un mismo país es que el análisis de las estructuras productivas, de la especialización regional y sus diferencias en relación con la media nacional, tiene gran interés. De hecho, la especialización regional puede constituir un factor explicativo del crecimiento económico regional (aunque considerando siempre el 'efecto nacional') y, por supuesto, de la posible convergencia de las regiones de un país en términos de productividad y de ingresos por habitante. 
La continua expansión de los servicios constituye una característica básica de las economías más desarrolladas. Incluso parecería que, antes o después, esto ocurrirá también en las economías menos avanzadas: la creciente demanda de servicios por parte de las empresas y la externalización de bastantes servicios que antes figuraban como parte de la producción industrial (o incluso de la agraria), junto con la también creciente demanda de servicios que realizan los individuos y las familias al aumentar su nivel de ingresos (Ley de Engel), contribuyen a explicar la terciarización de las economías, proceso al que también se une la expansión del comercio internacional de servicios.

Estos hechos han estimulado en los investigadores el interés por los servicios, lo que ha dado lugar a un notable aumento de las aportaciones sobre este tema en los últimos quince años en diversos países. De hecho, además de los análisis de carácter más macroeconómico, han aumentado los estudios referidos a ramas específicas de servicios. Y, por supuesto, también ha crecido el interés por el papel, los problemas y tendencias de los servicios desde la óptica territorial. De hecho, el proceso de terciarización y su reflejo a nivel regional, junto con las relaciones entre crecimiento y servicios a escala territorial, han sido objeto de un número creciente de aportaciones en los Estados Unidos, en varios países europeos y en América Latina (véase, por ejemplo, Chávez, 1995; Garza, 2006).

Este trabajo, que sigue a un estudio monográfico previo sobre los servicios y las regiones (Cuadrado \& Maroto, 2010), se inscribe en la línea de los estudios territoriales que se acaba de citar y su objetivo es abordar las relaciones entre los procesos de especialización productiva a escala regional en España, con particular énfasis en el caso de los servicios. El período que se toma como referencia es desde 1980 hasta 2006, lo cual está condicionado por la disponibilidad de una base de datos desagregada por sectores y regiones y de carácter oficial. Como se indicará más adelante, hubiera sido deseable incorporar al análisis los tres o cuatro últimos ejercicios, que han estado marcados por el impacto de la crisis financiera y económica internacional que ha afectado al país, pero no se dispone todavía de una información estadística homogénea como la aquí utilizada.

El trabajo pretende responder en alguna medida tres cuestiones: ¿ha tenido la especialización productiva un reflejo uniforme a escala regional?; ‘ hasta qué punto se ha reducido la desigualdad productiva a partir de 1980 y qué papel han desempeñado los servicios?; y, finalmente, ¿cabe afirmar que se ha producido, en realidad, una heterogeneidad de comportamientos? Dos hipótesis de partida sirvieron de guía para intentar dar respuesta a dichas preguntas. La primera es que el período estudiado parecería caracterizarse por una notable inercia en la especialización productiva de las regiones, lo que acaba determinando a su vez una baja convergencia de las estructuras productivas regionales. ${ }^{2}$ La segunda consiste en sostener que la especialización productiva influye en el crecimiento económico de las regiones y que los servicios han tenido, en particular, un claro papel en este sentido.

2 Este hecho no tiene por qué ser incompatible, por supuesto, con que las distintas regiones tengan comportamientos algo diferenciados. 
De acuerdo con este planteamiento y objetivos, el trabajo se organiza como sigue. Tras esta breve introducción, la segunda sección describe los datos y la metodología utilizados. En la tercera sección se realiza una primera aproximación al análisis de la especialización productiva y la terciarización de las regiones españolas a través de coeficientes de especialización. La cuarta sección se centra en analizar si se ha producido convergencia o divergencia en la evolución de las regiones en términos de estructura productiva y de especialización. Finalmente, la sección quinta cuantifica la contribución de los efectos de la estructura productiva y la localización geográfica en el crecimiento económico de las regiones españolas. También en este caso se presta particular atención a dichos efectos dentro del propio sector servicios. El artículo se cierra con una nota sobre cómo puede haber afectado la crisis a los resultados obtenidos en el análisis, y unas breves notas y observaciones finales.

\section{Datos y metodología}

\section{Base de datos}

La base de datos que se utiliza en este trabajo es la BD.MORES, base $2000,{ }^{3}$ lo cual marca una diferencia en relación con muchos trabajos y estudios regionales previos. Su elección se justifica porque permite estudiar, con datos homogéneos y de carácter anual, un período muy amplio (1980-2006, es decir, veintiséis años) de la evolución económica de las regiones españolas. Su principal ventaja en relación con la conocida serie BBVA es que, además de basarse directamente en las cifras estimadas oficialmente por el Instituto Nacional de Estadística (INE), ofrece datos anuales y no bienales, como esta última. Por otra parte, la posibilidad de utilizar los datos de la Contabilidad Regional ${ }^{4}$ del INE quedó también descartada, teniendo en cuenta los cambios metodológicos que se fueron introduciendo en la serie y la falta de enlaces que todavía subsiste en la misma.

Con todo, la base elegida tiene dos restricciones que es preciso señalar. La primera es que no permite una desagregación muy amplia del sector servicios. Solamente se diferencian cinco subsectores: Comercio y hostelería, Transportes y comunicaciones, Servicios de intermediación financiera, Otros servicios de mercado y Servicios no destinados a la venta. Esta desagregación condiciona en parte algunos de los resultados obtenidos, puesto que impide un análisis más profundo del grado de especialización por regiones, lo cual permitiría afinar los resultados que se obtienen al tener en cuenta, por ejemplo, datos estadísticos de las actividades del Comercio y la Hostelería por separado; o los correspondientes a las Telecomunicaciones, que ahora figuran junto con los Transportes; o cifras sobre la rama de Servicios a empresas, que en dicha base figuran integrados en Otros servicios de mercado.

3 Para más información, véase el documento metodológico de De Bustos et al. (2008). Agradecemos a los profesores J. Escribá y M. J. Murgui que nos anticiparan los datos básicos correspondientes a los años 2005 y 2006. No se han publicado todavía los datos posteriores.

4 "La Contabilidad Regional de España (CRE) es una operación estadística que el INE viene realizando desde el año 1980 y cuyo principal objetivo es ofrecer una descripción cuantificada, sistemática y lo más completa posible de la actividad económica regional en España (comunidades autónomas y provincias), durante el período de referencia considerado". En: www.ine.es $[$ N. de E.] 
El segundo problema de la base utilizada, actualmente de imposible superación, ${ }^{5}$ es que toma como año de partida 1980 y solo alcanza — cuando se realiza este trabajo- hasta 2006, lo que impide considerar los posibles cambios que se hayan producido posteriormente. Los dos ejercicios citados corresponden, por otra parte, a posiciones cíclicas bastante diferentes. En el primer caso (1980), la economía española estaba atravesando una fase de bajo crecimiento como consecuencia de la crisis económica internacional (crisis de la energía); en cambio, en el último ejercicio de la serie la economía española vivía un momento muy expansivo, que se prolongó todavía en 2007 y en parte de 2008, para caer después en una fase muy regresiva como consecuencia de la crisis financiera internacional y del hundimiento interno del sector construcción y de la actividad inmobiliaria. Dado el enfoque y el análisis adoptados en este trabajo, donde se toman en consideración todos los datos del período 1980-2006 y lo que se persigue es clarificar los principales cambios y las grandes tendencias que se han producido, se estima que la dispar situación del año de partida y del final no afectan, en lo esencial, la mayor parte de los resultados obtenidos. Estos reflejan las tendencias más profundas de los cambios estructurales producidos a nivel regional durante el extenso período estudiado, tendencias que son robustas y que no pueden haber cambiado sustancialmente más tarde, a pesar de que la crisis está implicando algunos cambios que se comentan más adelante.

\section{Metodología}

Desde el punto de vista metodológico, el análisis llevado a cabo se basa en la aplicación de varias técnicas. En una primera aproximación, el análisis de la especialización productiva se sirve de los conocidos coeficientes de especialización, que comparan el peso relativo de un sector dentro de una región en relación con la participación porcentual de dicho sector en el conjunto nacional. Una expresión genérica de dicho índice sería la siguiente:

$$
I E_{i j t}=\left[\frac{\xi_{i j}}{\sum_{i=1}^{n} \xi_{i j}} / \frac{\sum_{j=1}^{m} \xi_{i j}}{\sum_{i} \sum_{j} \xi_{i j}}\right]_{t=t_{k}}
$$

donde $i$ es el sector considerado, $j$ el indicativo regional, $\xi$ la variable objeto de y calculado respecto a un año concreto $t_{k}$. Este $I E_{i j t}$ siempre es positivo. Cuando supera la unidad, puede afirmarse que la región $j$ presenta especialización en el sector $i$ para el año $t$. En nuestro caso, la variable utilizada para el cálculo de estos indicado- 
res ha sido el empleo, con desagregación para las distintas comunidades autónomas (CC.AA.) españolas. ${ }^{6}$

Para completar la información que ofrece el indicador anterior, parece necesario analizar la intensidad del cambio en la especialización productiva regional con métodos más elaborados. A estos efectos se ha trabajado con un coeficiente de correlación no paramétrico, la rho de Spearman, cuya expresión es:

$$
\rho=1-\frac{6 \sum_{i=1}^{N} d_{i}^{2}}{N\left(N^{2}-1\right)}
$$

siendo $d_{i}$ las diferencias en el número de orden de las regiones (atendiendo al valor de su coeficiente de especialización) y $N$ el número de observaciones, en nuestro caso, las diecisiete CC.AA. Dicho coeficiente tiene un recorrido de $-1 \mathrm{a}+1$ y su signo indica la dirección de la relación, y el valor absoluto del índice indica la fuerza de la correlación entre las variables.

Con objeto de estudiar la evolución de la estructura productiva regional en su conjunto se ha utilizado el siguiente indicador de desigualdad en la estructura productiva:

$$
I D_{j}=\left[\frac{\sum_{i=1}^{m}\left(S_{i j}-\bar{S}_{i}\right)}{m}\right]^{1 / 2} ; \quad I \bar{D}=\frac{\sum_{j=1}^{17} I D_{j}}{17}
$$

donde $S_{i j}$ es la participación porcentual (en términos de empleo) del sector $j$ en la región $i$ y $\bar{S}_{i}$ es la participación porcentual del sector $i$ en la economía española; $m$ es el número de sectores analizados. El índice total $I \bar{D}$ se calcula como la media aritmética de los índices de cada una de las regiones analizadas. El valor del anterior índice sería cero si la estructura productiva fuera la misma en las $N=17$ regiones es-

6 La Constitución de 1978 permitió desarrollar en España un sistema político muy descentralizado que está constituido por diecisiete comunidades autónomas (regiones), a las que se suman las ciudades autónomas de Ceuta y Melilla, que en este trabajo están agregadas a Andalucía. Este sistema político ha supuesto una distribución de competencias desde la Administración Central a las administraciones autonómicas que, en muchos aspectos, supera claramente lo que es común en muchos estados federales.

7 Utilizado anteriormente, entre otros, por Raymond (1990); Garrido (2002); Cuadrado-Roura, García-Greciano y Raymond (1999); Cuadrado-Roura, Garrido y Mancha (2002); Cuadrado y Maroto (2009). 
pañolas. Como puede comprobarse, lo que hace el citado indicador no es otra cosa que medir el grado de dispersión media de una variable como es la participación sectorial en términos de empleo.

A la pregunta de si se ha producido (o no) una mayor igualdad en las estructuras productivas de las regiones en España también se puede responder con el indice de asociación geográfica tipo Florence, que permite cuantificar la diferencia o semejanza de las estructuras productivas comparándolas con la media nacional. Su expresión es la siguiente:

$$
I F_{j}=\frac{1}{2} \sum_{i=1}^{n}\left|\left(\frac{\xi_{i j}}{\sum_{i=1}^{n} \xi_{i j}}\right)-\left(\frac{\sum_{j=1}^{17} \xi_{i j}}{\sum_{i} \sum_{j} \xi_{i j}}\right)\right|
$$

donde $j$ es el indicativo de región, $i$ el de sector y $\mathbf{X}$ la variable objeto de estudio (empleo, en nuestro caso). El primer paréntesis identifica el peso (en términos de empleo) de un determinado sector $i$ sobre el total de la región, mientras que el segundo paréntesis plantea lo mismo con respecto al conjunto del país. Valores cercanos a cero indican estructuras sectoriales similares entre la región y el conjunto nacional, mientras que valores próximos a la unidad reflejarán estructuras sectoriales muy diferentes.

Un análisis alternativo y complementario al anterior se centraría en estudiar la evolución de la participación sectorial en cada región, no en términos comparativos, sino en relación con la propia economía regional. Para ello, en este trabajo se analiza el grado de concentración que tiene un sector dentro de una región, determinando, por tanto, su grado de diversidad o diversificación. Entre otras alternativas, se ha optado por una medida de diversidad basada en los indices de concentración industrial del tipo Hirschman-Herfindahl. El coeficiente de diversidad calculado tiene la siguiente expresión:

$$
C D_{j}=\sum_{i=1}^{n}\left(\frac{\xi_{i j}}{\sum_{j} \xi_{i j}}\right)^{2}
$$

El coeficiente será mayor cuanto menos homogénea sea la distribución sectorial del empleo dentro de una región. Es decir, cuanto más concentración sectorial se dé. Estos índices $C D_{j}$ nos ofrecen una imagen de la homogeneidad interna de cada región. 
Por último, para analizar la contribución sectorial al crecimiento regional se empleará una de las técnicas más extendidas en la literatura espacial: el análisis de estandarización shift-share (Peneder, 2002 y 2003; van Ark, 1995; Fagerberg, 2000; Timmer \& Szirmai, 2000; Maroto \& Cuadrado, 2007 y 2009 para datos nacionales; y Esteban, 2000; Paci \& Pigliaru, 1997, entre otros, a escala regional). ${ }^{8}$

El análisis shift-share convencional intenta cuantificar el crecimiento económico de una región a través de tres grandes efectos que tienen una naturaleza aditiva. El efecto nacional $(E N)$ muestra el nivel de producción o empleo alcanzado si la región hubiera registrado una tasa de crecimiento igual a la tasa de crecimiento nacional. La expresión de dicho efecto sería:

$$
E N=\sum_{i} \xi_{i j 0}\left(\frac{\sum_{i} \sum_{j} \xi_{i j t}}{\sum_{i} \sum_{j} \xi_{i j 0}}\right)-\sum_{i} \xi_{i j 0}
$$

donde $i$ es el indicativo de los sectores productivos, $j$ cada una de las regiones, y 0 y $t$ los dos momentos de tiempo contemplados en el análisis (1980 y 2006, en nuestro caso). El efecto neto total (ENT) es la cantidad resultante de la comparación entre el nivel de producción o empleo regional observado y aquel que se obtiene aplicando la tasa de crecimiento nacional. Por tanto, esta cantidad puede ser positiva (si la región ha crecido más que la media nacional) o negativa (en caso contrario). Su expresión es la siguiente:

$$
E N T=\sum_{i} \xi_{i j t}-\sum_{i} \xi_{i j 0}\left(\frac{\sum_{i} \sum_{j} \xi_{i j t}}{\sum_{i} \sum_{j} \xi_{i j 0}}\right)
$$

Este efecto neto puede descomponerse, como es sabido, en el efecto proporcional y el efecto diferencial. El efecto proporcional $(E P)$ recoge las diferencias existentes entre una región y la media debidas a la especialización productiva, razón por la que se le conoce también como efecto estructura o industry mix. Su cálculo se realiza atendiendo a la siguiente expresión:

8 Esta metodología presenta algunas ventajas bien conocidas, pero también ha sido objeto de críticas, basadas principalmente en su uso como herramienta predictiva, la estabilidad de los resultados ante distintas agregaciones, o ciertos problemas de asimetría en la estimación. 
A su vez, el efecto diferencial ( $E D)$, denominado también efecto competitivo o regional, cuantifica la influencia sobre el crecimiento regional de factores de diversa naturaleza. Para estimarlo, se comparan las tasas regionales de crecimiento de cada uno de los sectores productivos. Su expresión es:

$$
E P=\sum_{i} \xi_{i j 0}\left[\left(\frac{\sum_{j} \xi_{i j t}}{\sum_{j} \xi_{i j 0}}\right)-\left(\frac{\sum_{i} \sum_{j} \xi_{i j t}}{\sum_{i} \sum_{j} \xi_{i j 0}}\right)\right]
$$

De acuerdo con esta descomposición, una región puede estar situada en seis tipologías o categorías distintas, tres con ENT positivo y tres con ENT negativo (Cuadro 1).

CUADRO 1 | Tipologías regionales según análisis del signo de los factores explicativos del crecimiento regional (shift-share)

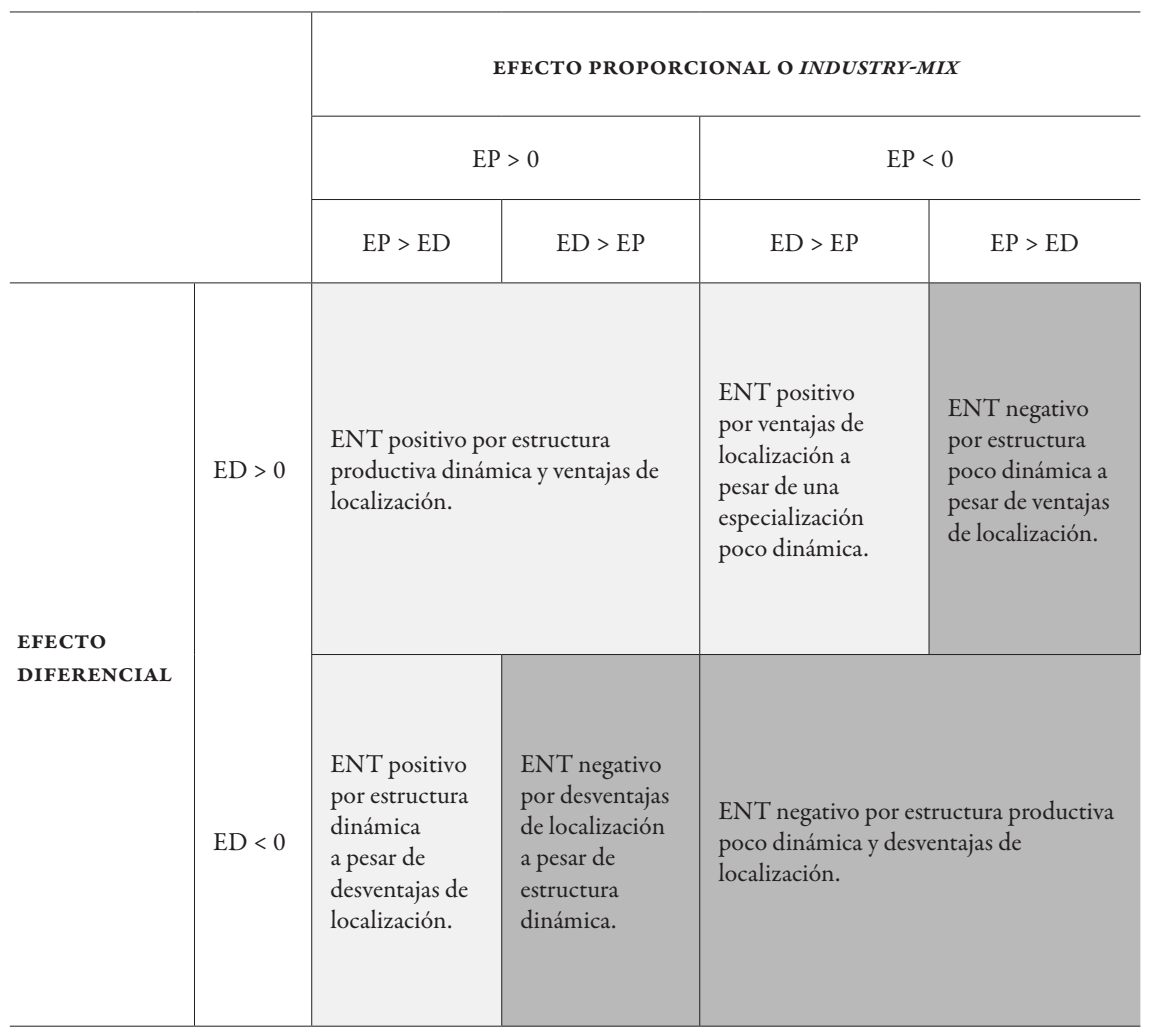

FUENTE ELABORACIÓN PROPIA.

NOTA: EN GRIS OSCURO, TIPOLOGÍAS REgIONALES CON ENT < 0; EN GRIS CLARO, TIPOLOGÍAS REGIONALES CON $\mathrm{ENT}>0$. 


\section{'Terciarización' de las regiones: una aproximación a través de indicadores de especialización y localización}

Una primera aproximación a la evolución de la especialización regional que nos permita contrastar la primera de las hipótesis establecidas puede lograrse estimando los coeficientes de especialización, que comparan el peso relativo de una determinada actividad económica dentro de una región en relación con la participación porcentual de la misma en el conjunto de la economía; en nuestro caso, España.

La característica más general que se extrae de resultados obtenidos al considerar los cinco grandes sectores productivos es que, en el caso español, la especialización regional muestra una clara inercia temporal. Si tomamos como referencia el valor unitario del coeficiente, los resultados indican que son muy pocas las regiones que se especializan o des-especializan significativamente a lo largo del período 1980-2006. Salvo en el caso de la construcción, en los demás grandes sectores productivos (agricultura, industria y energía, y servicios) la proporción de regiones que presentan alguno de estos dos tipos de comportamiento - especialización/ des-especialización - siempre se sitúa por debajo del 20\%. Lo mismo ocurre al considerar con detalle las distintas actividades de servicios, excepto en el caso de los Transportes y comunicaciones, donde el porcentaje de regiones que se des-especializa es más destacable durante el período estudiado. Por el contrario, el refuerzo o la atenuación de la especialización y, sobre todo, la ausencia de dicha especialización es una característica bastante común.

Sin embargo, hay que tener en cuenta dos observaciones. En primer lugar, como ya se anticipó en el apartado anterior, está el reducido nivel de desagregación con que se ha operado para realizar estos cálculos, ya que para que los cambios pudieran registrarse mejor, sería preciso disponer de datos por ramas de actividad mucho más desagregados. Este hecho es particularmente importante en el caso del sector servicios, puesto que en él coexisten ramas de actividad muy heterogéneas entre sí. Y, por otra parte, hay que recordar que el análisis citado se efectúa en términos relativos. Esto supone que una región aumenta o disminuye su grado de especialización en función de lo que hace el resto de las regiones y no en función de si dicha actividad económica gana o pierde peso dentro de la región estudiada. Lo cual, si bien resulta útil para realizar comparaciones estáticas, puede constituir un obstáculo a la hora de analizar cambios temporales, ya que podría esconder algunas variaciones interesantes, aunque no llegaran a constituir grandes tendencias.

\section{La especialización regional y los servicios}

Como es lógico, los servicios han incrementado su peso en todas las CC.AA. españolas, siguiendo la tendencia general de la economía del país durante el período estudiado (véanse Cuadrado, 2008; y Cuadrado \& González, 2009). Pero, desde la óptica regional, hay que subrayar sobre todo que existe una notable inercia en los cambios, a pesar de que se registran algunas variaciones de interés. Dicha inercia se observa cuan- 
do se analiza el coeficiente de correlación entre los índices de especialización sectorial (tomando todas las ramas de actividad) de las regiones españolas a principios de los ochenta y en el año 2006, cuyo valor es de 0,78 (con un $p$-valor de 0,000 ).

A principios de los ochenta, las regiones que lideraban el ranking de especialización en actividades de servicios eran Madrid, Canarias, Baleares, Andalucía y Cataluña, mientras que Galicia, La Rioja, Asturias, Castilla y León y Castilla-La Mancha presentaban los peores coeficientes de especialización terciaria. 9 Pues bien, en 2006, al cerrar el extenso período estudiado, las cinco primeras regiones del ranking seguían siendo prácticamente las mismas, si bien Asturias desplazaba a Cataluña. Por su parte, en dicho año las regiones con menos especialización en servicios son también las mismas que en 1980. Solo Asturias asciende a los primeros puestos por su creciente especialización en servicios, debida en buena parte al retroceso industrial y agrario que esta región ha experimentado en los veintiséis años analizados. Ahondando en esta idea, el coeficiente de correlación de los rankings de especialización en servicios entre 1980 y 2006 es de 0,77 (con un p-valor de 0,000).

Con objeto de contrastar la primera de las hipótesis presentadas en este trabajo - sobre la inercia en la especialización productiva - se ha llevado a cabo el contraste no paramétrico de la $Z$ introducido por Brockett, Golany y Li (1999) a partir del trabajo de Brockett y Kemperman (1980). La hipótesis nula, según el contraste introducido por estos autores, es que el vector de los $k$ rangos para cada unidad de decisión no depende del tiempo, es decir, que $\operatorname{los} C_{1 j} C_{2 j} \ldots, C_{k j}$ rangos se distribuyen uniformemente a lo largo de las $k$ ! posibles ordenaciones de $(1,2, \ldots, k)$. A partir de este análisis, se puede construir el estadístico $S=\sum_{j=1}^{n} \sum_{t=1}^{k} t \cdot C_{t j}$, , cuya distribución sigue una normal de media $\quad \mu=E(S)=\frac{n \cdot k}{4}(k+1)^{2} \quad \mathrm{y}$ varianza, $\quad \sigma^{2}=\operatorname{Var}(S)=\frac{n}{144} \cdot k^{2}\left(k^{2}-1\right)(k+1)-\frac{k(k+1)}{144} \sum_{j=1}^{n} \sum_{r}\left(d_{j r}^{3}-d_{j r}\right)$, siendo $d_{j r}$ el número de valores $h_{i j}, \cdots, h_{k j}$ que empatan dentro del $r$-ésimo grupo de empates. Finalmente, se normaliza el anterior estadístico $S$ en una distribución normal estándar, utilizando para ello el estadístico $Z=(S-\mu) / \sigma$. La hipótesis nula de que no existe una tendencia significativa en los rangos de especialización observados puede rechazarse a un nivel de significación $\alpha$ cuando $Z \leq-Z_{\alpha / 2}$ o $Z \geq Z_{\alpha / 2}$ , donde $Z_{\alpha / 2}$ denota el percentil superior de la distribución normal que deja a la derecha una probabilidad de $\alpha / 2$. El resultado para las regiones españolas en el período considerado muestra un estadístico $Z=0,64$ ( $\mathrm{p}$-valor $=0,74$ ), por lo que la hipótesis de no tendencia en la especialización productiva puede ser aceptada.

Si del análisis del sector servicios como agregado se pasa al estudio de la especialización regional 'dentro' del propio sector, los resultados que se obtienen no

9 En el anexo se ha incluido un mapa en blanco (Figura A-1) que permite identificar la situación de las distintas CC.AA. en la geografía española. 
difieren sustancialmente de los del conjunto: la inercia temporal relativa en la especialización regional y los cambios en la estructura productiva del sector terciario son similares a los observados previamente. Sin embargo, el análisis da cuenta de algunos procesos de especialización regional interesantes, como los que han registrado varias regiones en el caso del Comercio y la hostelería (en Asturias y Cantabria, p. ej.) o en los Transportes y comunicaciones (Baleares, Canarias, Madrid), a los cuales se suma el incremento del peso de los Servicios no destinados a la venta $(\mathrm{SNDV})^{10}$ en la estructura de algunas regiones (Extremadura, Castilla-La Mancha), al que más tarde nos referiremos. Hay que tener en cuenta, en todo caso, que este análisis se efectúa en términos relativos y que, por tanto - como ya se ha indicado-, una región aumenta o disminuye su especialización en función de lo que hace el resto de regiones y no debido a que dicha actividad económica simplemente gana o pierde peso dentro de la región analizada.

\section{Intensidad del cambio en términos de especialización}

Para complementar y dejar más claro el proceso de especialización productiva regional, parece necesario analizar el grado o la intensidad de los cambios registrados, utilizando para ello otros métodos alternativos. A estos efectos, se ha trabajado con un coeficiente de correlación no paramétrico: la rho de Spearman. Los resultados que proporciona este indicador muestran que el cambio en la especialización regional ha sido de distinta intensidad por sectores. En el sector agrario, el valor de la rho de 0,831 indica una inercia temporal muy fuerte, de tal forma que el ranking regional apenas experimentó modificaciones durante el período estudiado. Conclusiones similares se extraen en los casos de la minería y extracción $(0,737)$ y en las manufacturas $(0,872)$. El sector servicios tampoco se aparta mucho de los anteriores, si bien el coeficiente es más reducido $(0,647)$, lo que sugiere que se produjo un mayor cambio comparativo. Por último, la construcción es el sector donde se detecta más movilidad, si bien sin una dirección clara $(-0,002)$. Su dinámica está vinculada, sin duda, al papel que este sector desempeñó en la expansión de la economía española, con un reflejo particularmente importante en las regiones de la costa mediterránea, pero también en Madrid y en algunas provincias del interior de España.

Los coeficientes estimados permiten avanzar algunas ideas sobre los distintos patrones de especialización de las regiones españolas, tanto desde el punto estático como en cuanto al cambio estructural observado. Es obvio que una región no puede estar especializada en todos los sectores a la vez, pero lo relevante de los indicadores anteriores es mostrar que aquellas que están especializadas en actividades de servicios no lo están ni en actividades primarias ni en las manufactureras y que dicho patrón se mantuvo bastante estable desde principios de los años ochenta hasta 2006.

Como segunda aproximación se analizó la intensidad de los cambios estructurales experimentados en la composición del sector servicios en las distintas regiones

10 Como es sabido, en este sector se integran todos aquellos servicios que son ofrecidos por el sector público, ya sean la educación y la sanidad públicas, los servicios sociales o las actividades de las propias Administraciones Públicas (Estado, regiones y municipalidades). 
españolas, a cuyo efecto se estimaron también los coeficientes rho de Spearman. Los valores obtenidos para el conjunto figuran en el Cuadro 2, cuyas cifras permiten subrayar varios rasgos relevantes.

CUADRo 2 | Coeficiente rho de Spearman. Especialización regional en términos de empleo, 1980-2006

\begin{tabular}{|c|c|c|c|c|c|c|c|c|c|c|}
\hline & CYH_8O & TYC_8O & FIN_80 & OTR_80 & NO_8o & CYH_O6 & TYC_06 & FIN_06 & OTR_06 & NO_06 \\
\hline CYH_8O & 1,0000 & 0,1225 & 0,3333 & 0,4265 & 0,1716 & 0,5319 & 0,2255 & 0,0025 & 0,2451 & $-0,2549$ \\
\hline TYC_80 & & 1,0000 & 0,1691 & 0,5147 & 0,4706 & 0,3824 & 0,6250 & $-0,0858$ & 0,4828 & 0,2598 \\
\hline FIN_80 & & & 1,0000 & 0,6887 & 0,0000 & 0,0000 & 0,3848 & 0,7230 & 0,5858 & $-0,4020$ \\
\hline OTR_80 & & & & 1,0000 & 0,1373 & 0,5343 & 0,6838 & 0,4583 & 0,7574 & $-0,2034$ \\
\hline NO_80 & & & & & 1,0000 & $-0,1054$ & 0,1103 & $-0,1127$ & 0,1078 & 0,4485 \\
\hline CYH_O6 & & & & & & 1,0000 & 0,5319 & $-0,0564$ & 0,4877 & 0,0490 \\
\hline TYC_06 & & & & & & & 1,0000 & 0,1716 & 0,7966 & $-0,0711$ \\
\hline FIN_06 & & & & & & & & 1,0000 & 0,3971 & $-0,1250$ \\
\hline OTR_06 & & & & & & & & & 1,0000 & $-0,3137$ \\
\hline NO_o6 6 & & & & & & & & & & 1,0000 \\
\hline
\end{tabular}

FUENTE ELABORACión PROPIA.

NOTA: CYH = COMERCIO Y HOSTELERÍA; TYC = TRANSPORTES Y COMUNICACIONES; FIN = INTERMEDIACIÓN FINANCIERA; OTR = OTROS SERVICIOS DE MERCADO; NO = SERVICIOS NO DESTINADOS A LA VENTA.

En cuanto a los subsectores de servicios, el mayor coeficiente rho corresponde a Otros servicios de mercado $(0,7574)$ y a los servicios de Intermediación financiera $(0,723)$, mientras que las inercias temporales tienen menor intensidad en Transportes y comunicaciones $(0,625)$, Comercio y hostelería $(0,532)$ y, fundamentalmente, en los Servicios no destinados a la venta $(0,448)$. Por otra parte, las interrelaciones temporales entre algunas actividades de servicios y el resto de los servicios ofrecen como resultado que los SNDV muestran una correlación negativa con el Comercio y la hostelería y con los Servicios financieros, mientras que el resto de las asociaciones son positivas, aunque su intensidad no sea muy elevada. Esto sugiere que se han producido algunos procesos de sustitución en la especialización regional en las distintas ramas terciarias. En concreto, los datos obtenidos indican, por ejemplo, que la mayor terciarización de algunas regiones en SNDV determinó un retroceso relativo de algunos servicios de mercado, especialmente los financieros, el comercio y la hostelería.

A partir de los resultados obtenidos mediante los cálculos realizados en esta sección, es posible extraer algunas conclusiones. En primer lugar, que los patrones de especialización regional, lejos de tener un comportamiento convergente -es decir, tendientes a la igualación en sus estructuras productivas- han permanecido relativamente estables en el tiempo, con una movilidad interna muy reducida, pese a que el análisis corresponde a un período de más de cinco lustros. Al cerrar el período, las regiones más especializadas en servicios son Madrid, Baleares, Canarias y Cataluña. En el primer caso, el peso de los servicios se debe a subsectores como 
Intermediación financiera, Transportes y comunicaciones y los Servicios a las empresas, encuadrados en este caso dentro de Otros servicios de mercado. En Cataluña, además de estos, también tienen una importancia significativa los servicios de Hostelería. Esta actividad tiene singular importancia en Canarias y Baleares, donde el desarrollo del turismo ha impulsado el elevado predominio de los servicios en su estructura productiva.

Por último, conviene destacar también el comportamiento de los SNDV, ya que varias de las CC.AA. no solo han mantenido una elevada especialización en este tipo de servicios, sino que incluso la han aumentado durante el período estudiado. Esto ha sucedido, en particular, en Extremadura, Murcia, Andalucía y Madrid; al mismo tiempo, algunas regiones que no estaban especializadas en este tipo de servicios han pasado a estarlo bastante más, como sucede con las dos Castillas, Galicia, Asturias y Aragón. Estos hechos guardan relación, sin duda, con el desarrollo de lo dispuesto en la Constitución de 1978, que ha supuesto la creación de estructuras de Gobierno y de Administración propias en todas las regiones. Tal cambio ha implicado la transferencia de funcionarios a las regiones y la expansión de sus estructuras administrativas, que han asumido competencias en educación, sanidad y otros servicios públicos, y además incrementaron su peso en toda España gracias al desarrollo del Estado de bienestar. El aumento del peso de los SNDV ha sido particularmente destacable en las regiones cuyo nivel de desarrollo era y es comparativamente más bajo (PIB por habitante inferior a la media nacional). Todas las regiones indicadas, con la excepción de Madrid, tienen esta característica. Por el contrario, en las regiones con un mayor grado de desarrollo, la terciarización fue protagonizada por los servicios de mercado, aunque los SNDV también crecieron en términos absolutos.

\section{Especialización productiva y convergencia en las estructuras productivas de las regiones en España}

Los resultados obtenidos hasta ahora sugieren que, en el período estudiado, no se produjo una igualdad creciente en las estructuras productivas de las regiones, sino que los cambios registrados dejaron a las regiones en posiciones parecidas a las que ya tenían al comienzo del período estudiado. Sin embargo, es preciso confirmar (o no) esta presunción.

Para calcular el grado de desigualdad existente entre las estructuras productivas regionales y su evolución en el tiempo, se ha utilizado el indicador de desigualdad definido anteriormente. El Gráfico 1 muestra los resultados obtenidos al estimar dicho indicador. La línea continua de dicha figura indica que, en términos de empleo, la estructura productiva de las regiones españolas se hizo progresivamente algo más homogénea durante el período analizado. El avance hacia una relativa mayor homogeneidad es más marcado entre 1980 y 1994, ya que prácticamente se detiene en los años siguientes. La razón de lo que sucede en la citada primera fase radica, esencialmente, en la fuerte pérdida de peso que registra el sector primario en las regiones 
más atrasadas. Este hecho contribuye a explicar la evolución de la sigma convergencia en términos de productividad laboral hasta principios de los noventa, ${ }^{11}$ y por ende, la convergencia en términos de ingresos per cápita. En la Figura 1 también se observa una ligera convergencia en los valores de la distribución (cuartiles), sobre todo hasta mediados de los noventa.

GRÁFICO 1 | Evolución del índice de desigualdad de la estructura productiva regional en España 1980-2006, en términos de empleo

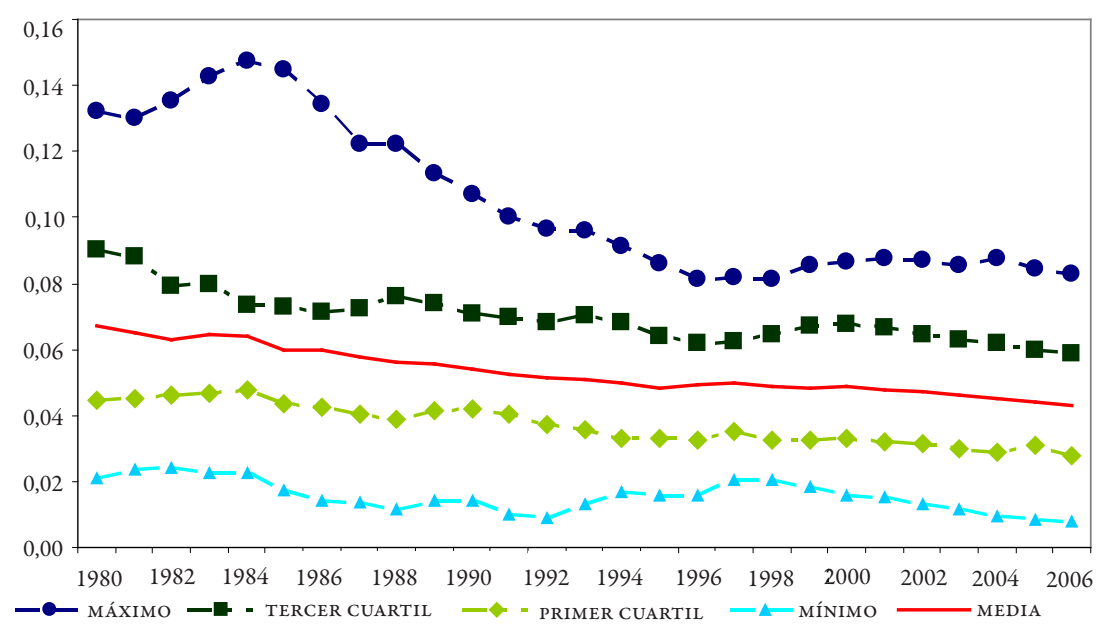

FUENTE ELABORACIÓN PROPIA A PARTIR DE BD.MORES 2000.

Sin embargo, a partir de mediados de los noventa, la convergencia en términos de estructuras productivas regionales se ralentizó sustancialmente. Las diferencias interregionales que entonces existían en cuanto al peso de las manufacturas y los servicios eran ya muy bajas, y prácticamente inexistentes en el caso del sector construcción, mientras que continuaron reduciéndose cada vez más en el caso del sector agrario. El resultado final es que en los últimos años las diferencias estructurales se han mantenido muy bajas (en torno a 0,05 ), con lo que el margen para posibles efectos positivos por esta vía en el futuro (cambio estructural) parece muy poco probable. La conclusión que se obtiene a partir de los datos anteriores es que hasta el primer tercio de los años noventa se produjo una tendencia convergente en términos de empleo, aunque la dispersión en esta variable es mayor que en términos de producción. Además, la reducción de la desigualdad en este indicador implica,

11 Este hecho se estudia más ampliamente en Cuadrado y Maroto (2010b). El trasvase de mano de obra desde los sectores menos productivos (el agrario, entre ellos) hacia sectores con una productividad más alta permite incrementar la productividad laboral total, debido en gran medida a los cambios estructurales de la economía, aunque la productividad también mejora en cada sector y/o rama de actividad a través de la innovación, el progreso tecnológico y la capitalización. 
como condición necesaria, que se produzca una igualación intrasectorial de las estructuras productivas regionales. Es decir, se ha de verificar que se produce una convergencia $\beta$ o, dicho de otra forma, una relación negativa entre la especialización sectorial y la tasa de crecimiento relativo.

El Gráfico 2 muestra la convergencia regional en términos de empleo con referencia al sector servicios. Los resultados de nuestro análisis indican que los procesos de convergencia son más pronunciados en el caso de los servicios que en los demás sectores. Cabe afirmar, incluso, que las estructuras productivas, al menos en términos de empleo y con referencia a los servicios, han tendido a igualarse en el período 1980-2006.

GRÁFICO 2 | Convergencia en el sector servicios. Empleo, 1980-2006

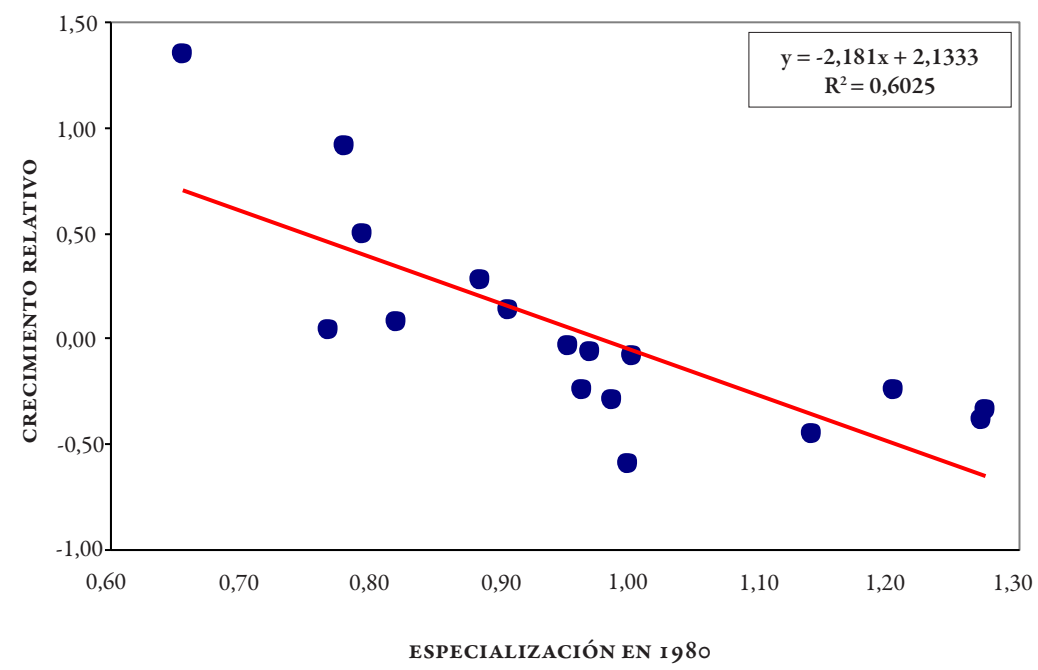

FUENTE ELABORACIÓN PROPIA. DATOS BD.MORES 2000.

NOTA AJUSTE LINEAL DEL TIPO $\frac{d L_{i j t}}{d t}=\alpha+\beta L_{i j 1980}$ DONDE $L_{i / T}$ SON LOS ÍNDICES DE ESPECIALIZACIÓN Y SU DERIVADA ES LA TASA DE CRECIMIENTO ACUMULATIVA ANUAL.

No hay que olvidar, sin embargo, que un proceso convergente de especialización puede ser compatible con un aumento en la desigualdad regional, y viceversa. La importancia relativa de cada sector económico en cada economía regional puede dar como resultado un mayor grado de desigualdad si dichas actividades ganan peso en el transcurso del tiempo. En consecuencia, la pregunta que cabe plantearse es si los procesos de terciarización observados permiten afirmar que se ha producido (o no) una mayor igualdad en las estructuras productivas de las regiones en España. Para responder a esta cuestión se ha elaborado un índice de asociación geográfica tipo Florence, que permite cuantificar la diferencia o semejanza de las estructuras productivas intersectoriales de las distintas CC.AA., comparándolas con la media nacional. Los resultados obtenidos se sintetizan en el Cuadro 3. 
CUADRO 3 | Índice de Florence de la estructura productiva regional en España

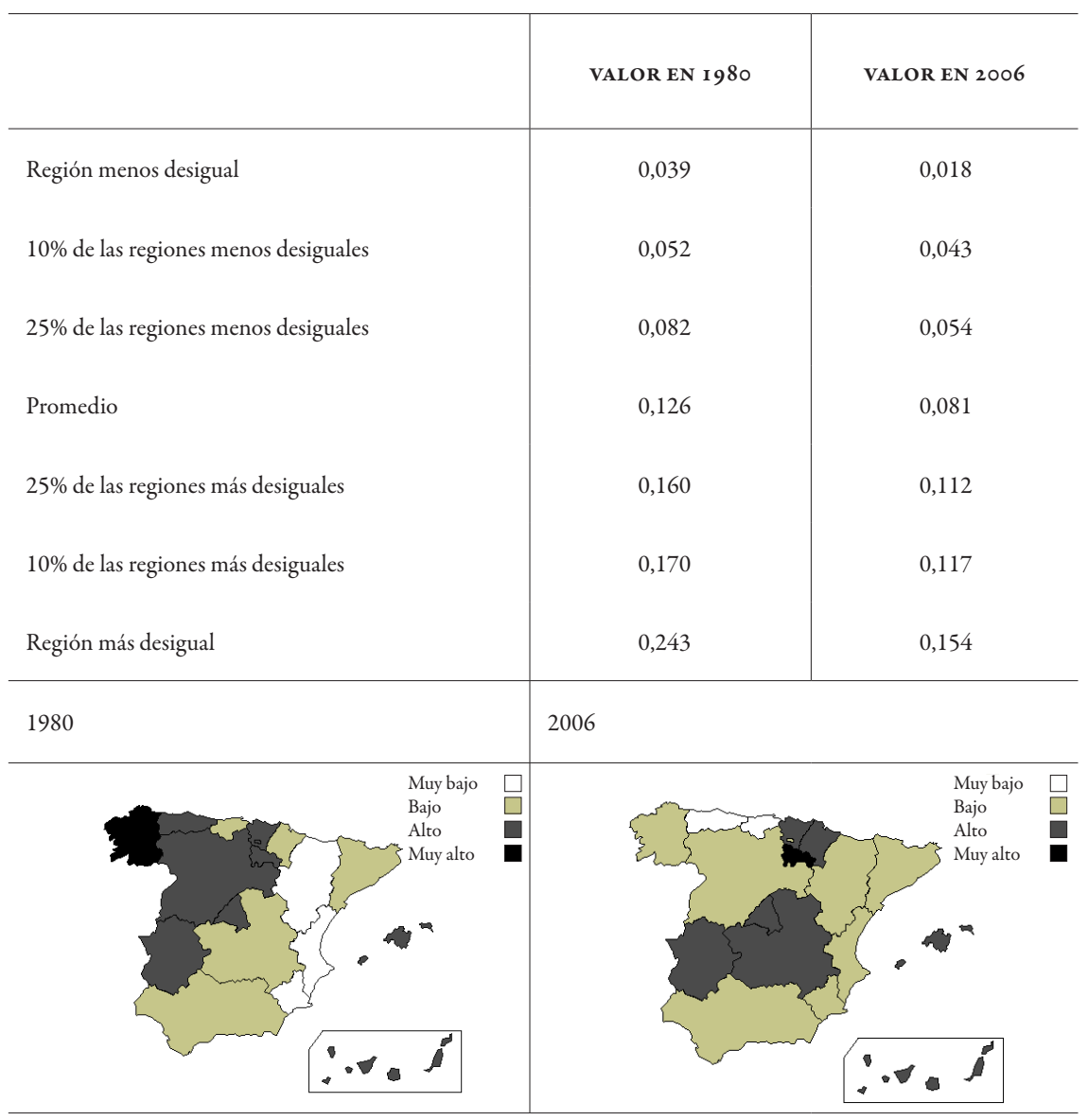

FUENTE ELABORACIÓN PROPIA. DATOS BD.MORES 2000.

La evolución que registra dicho índice permite subrayar varios hechos. En primer lugar, que existe una gran discrepancia entre los valores extremos: entre la región que en 1980 tenía una estructura productiva más cercana a la media nacional (Aragón) y la que entonces se alejaba más de ella (Galicia) existe una diferencia de 0,20 en sus respectivos $I F$. El coeficiente disminuye a lo largo del período estudiado hasta situarse, en 2006, en un valor de 0,13, y las regiones menos y más desiguales son ahora Asturias y La Rioja, respectivamente. Segundo, en la mayoría de los casos el índice de Florence estimado para cada región disminuye desde 1980 hasta 2006. Esto indica que las estructuras productivas sectoriales han ido aproximándose con el paso del tiempo. Únicamente Murcia, Navarra y, ligeramente, Castilla-La Mancha han observado un incremento en sus índices, alejándose así de la estructura promedio del conjunto español. 
GRÁfico 3 | Evolución del índice de Florence, 1980-2006

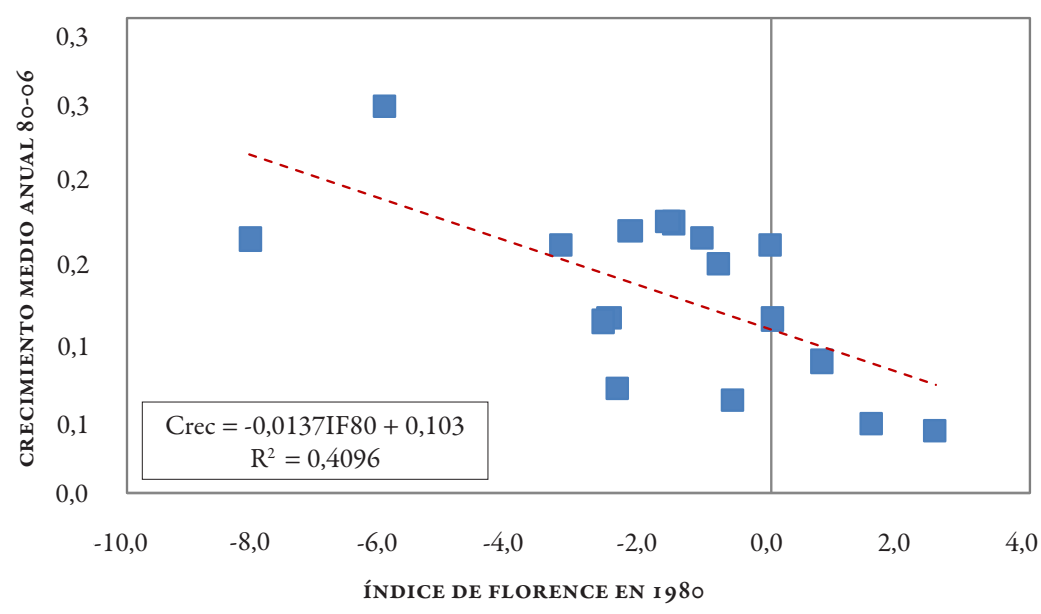

FUENTE ELABORACIÓN PROPIA. DATOS BD.MORES 2000.

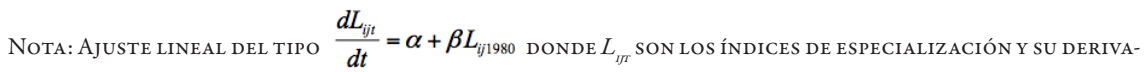
DA ES LA TASA DE CRECIMIENTO ACUMULATIVA ANUAL.

Sin embargo, confirmando lo planteado con respecto a la terciarización de las regiones, la regresión muestra que dicha convergencia tiene una significatividad bastante limitada. A pesar de que existe una relación negativa entre el índice inicial en 1980 y la tasa de crecimiento (que indica que a estructuras más desiguales en 1980, mayor ha sido la aproximación en la estructura productiva), el $\mathrm{R}^{2}$ obtenido es bastante bajo. $\mathrm{Y}$ debe tenerse en cuenta, además, que el comportamiento de dos regiones -Asturias y Galicia - influye en gran medida en la relación negativa obtenida.

Los mapas que figuran en la parte inferior del Cuadro 3 muestran la evolución territorial en términos de igualdad o desigualdad de las estructuras productivas regionales en España. Mientras que las CC.AA. con una estructura más parecida a la media nacional a principios de los ochenta eran Aragón, la Comunidad Valenciana y Murcia, la estructura productiva de dichas regiones se ha ido alejando de la española, y Asturias y Cantabria son en 2006 las que menores índices de desigualdad presentan. En el extremo opuesto, Galicia, la región con una estructura más desigual en 1980, registra un proceso de ligera convergencia hasta 2006.

Por lo tanto, la principal conclusión que puede obtenerse es que, pese a la existencia de cierto patrón de convergencia en términos de estructuras productivas, este proceso de convergencia no ha sido homogéneo, ni se ha mantenido en el tiempo durante los últimos veintiséis años. En el conjunto de las regiones, la concentración sectorial es ligeramente mayor en $2006(0,0319)$ que a principios de los ochenta $(0,0316)$, como muestra la evolución del promedio total. La región más diversificada (con me- 
nor concentración) es La Rioja, tanto en 1980 como en 2006, mientras que Cataluña (1980) y Andalucía (2006) figuran como las menos diversificadas. En promedio, el sector servicios explica en 2006 el 21\% del valor del indicador de diversidad, porcentaje que crece a partir de 1980 como consecuencia lógica de los procesos de terciarización que experimentan las regiones españolas durante el período estudiado.

CUADRO 4 | Coeficiente de diversidad de la estructura productiva regional en el sector servicios (y su distribución porcentual por actividades)

\begin{tabular}{|c|c|c|c|c|c|c|}
\hline & $\begin{array}{l}\text { SECTOR } \\
\text { SERVICIOS }\end{array}$ & $\begin{array}{l}\text { COMERCIO Y } \\
\text { HOSTELERÍA }\end{array}$ & $\begin{array}{l}\text { TRANSPORTE Y } \\
\text { COMUNICACIONES }\end{array}$ & FINANZAS & $\begin{array}{l}\text { OTROS SERVICIOS } \\
\text { DE MERCADO }\end{array}$ & $\begin{array}{l}\text { SERVICIOS } \\
\text { FUERA DE } \\
\text { MERCADO }\end{array}$ \\
\hline \multicolumn{7}{|l|}{1980} \\
\hline PROMEDIO & 0,0341 & 17,8 & 19,0 & 22,5 & 22,1 & 18,5 \\
\hline $\begin{array}{l}\text { IO\% MÁS } \\
\text { DESIGUALES }\end{array}$ & 0,1313 & 21,0 & 24,0 & 15,8 & 19,1 & 20,1 \\
\hline IO\% MÁS IGUALES & 0,0011 & 15,3 & 21,5 & 22,9 & 22,0 & 18,2 \\
\hline \multicolumn{7}{|l|}{2006} \\
\hline PROMEDIO & 0,0354 & 18,1 & 20,2 & 22,0 & 21,5 & 18,2 \\
\hline $\begin{array}{l}\text { IO\% MÁs } \\
\text { DESIGUALES }\end{array}$ & 0,1308 & 21,1 & 18,9 & 16,6 & 19,0 & 24,4 \\
\hline I $\%$ MÁS IGUALES & 0,0008 & 19,1 & 19,9 & 20,0 & 22,8 & 18,2 \\
\hline
\end{tabular}

FUENTE ELABORACIÓN PROPIA.

Un paso más en el análisis consiste en examinar qué sucedió dentro del sector servicios. A tal efecto se han estimado los índices de concentración tipo Hirschman-Herfindahl, cuyos resultados se sintetizan en el Cuadro 4. Los datos muestran que, como sucede en el conjunto de sectores productivos (Cuadrado \& Maroto, 2010a), también dentro del sector servicios la diversidad productiva disminuye ligeramente durante el período 1980-2006 (el coeficiente asciende de 0,0341 a $0,0354)$ o, en otras palabras, que se produce una ligera mayor concentración. Esto último se debe, esencialmente, al comportamiento de las regiones intermedias y no a las de los extremos. Tanto en las regiones más desiguales como en las más diversificadas, el coeficiente de diversificación disminuye ligeramente en el período analizado (de 0,1313 al principio a 0,1308 , y de 0,0011 a 0,0008 , respectivamente). La Rioja vuelve a ser la región más diversificada de España en cuanto al sector servicios, mientras que Cataluña (1980) y Madrid (2006) son las menos diversificadas.

Por último, los datos anteriores muestran que los subsectores que han tendido a concentrarse más son Intermediación financiera y Otros servicios de mercado (alrededor del 22\%). Por su parte, Transportes y comunicaciones contabilizan en torno al 20\% de la concentración terciaria en las regiones españolas; y el 36\% restante se reparte entre Comercio y hostelería y los SNDV. Otro hecho destacable es que estas contribuciones subsectoriales permanecen muy estables entre 1980 y 2006. 


\section{Especialización productiva y crecimiento de los servicios: una aproximación cuantitativa a través de técnicas de descomposición}

En la sección anterior se han puesto de manifiesto las principales características de las estructuras productivas regionales a través de la medición de la especialización. En esta, el objetivo es confirmar (o no) la segunda hipótesis del trabajo, mostrando el grado de influencia de la especialización productiva en el crecimiento económico de las regiones, con especial atención al papel desempeñado por la expansión de los servicios.

Como es sabido, a lo largo del período 1980-2007, y muy especialmente a partir de mediados de la década de los noventa, el PIB español registró una de las fases de crecimiento más elevado de su historia. De hecho, la comparación entre las tasas de crecimiento de España y de la Unión Europea-15 muestra que la economía española creció prácticamente siempre por encima de la media comunitaria, superando al menos en un punto porcentual dicha media. Este fuerte crecimiento estuvo acompañado en los últimos años por una intensa creación de empleo, aunque con tasas de productividad muy reducidas, o a veces nulas (Maroto \& Cuadrado, 2006 y 2008; Segura, 2006; Domenech, 2008). Este elevado crecimiento se interrumpió drásticamente en 2008 como consecuencia de la crisis financiera internacional, pero también gracias al derrumbe de los dos sectores que habían sido los principales protagonistas de la expansión española: la construcción (y la actividad inmobiliaria vinculada a la vivienda) y el consumo interior.

Desde la perspectiva regional, todas las CC.AA. siguieron la trayectoria expansiva registrada por la economía española, aunque las tasas regionales de crecimiento año a año muestran diferencias apreciables. La nota común durante el período 1980-2006 fue la reducción de las diferencias entre las regiones que más crecieron y las que obtuvieron peores registros. El Cuadro 5 ofrece datos sobre algunas variables regionales clave durante el período, como son la tasa de crecimiento medio del PIBpm (Producto Interior Bruto a precios de mercado) y las tasas de variación de la población y del empleo. Figuran asimismo en dicho cuadro las variaciones que experimentan las diecisiete CC.AA. en cuanto a su peso en el conjunto español, tanto en PIBpm, como en población y empleo total.

Como es obvio, las explicaciones que cabe ofrecer en relación con lo que muestran los datos relativos al crecimiento regional son bastante variadas. Una causa es siempre, sin duda, el comportamiento global de la economía nacional: si esta crece, la mayoría de las regiones lo hace también, aunque no de forma homogénea. En este sentido, hay coincidencia en afirmar que la especialización productiva de cada región puede ser una de las causas fundamentales de que su crecimiento esté por encima o por debajo de la media de crecimiento de una economía nacional (Combes \& Overman, 2003; Ezcurra, Pascual \& Rapún, 2006; Hallet, 2000; y Cuadrado \& Maroto, 2008). Las regiones especializadas en actividades más dinámicas registrarán tasas de crecimiento claramente superiores a las tasas medias de crecimiento del país. Lo contrario también suele ser cierto: las ramas menos dinámicas y expansivas lastran el crecimiento de las regiones que están más especializadas en ellas. Con 
todo, esta interpretación tampoco puede ser tan simple, ya que la composición por ramas de actividad de cualquier economía regional puede dar lugar a que lo que unas aportan se compense con el bajo crecimiento de otras.

CUADro 5 | Variaciones del PIBpm, la población y el empleo por CC.AA., 1980-2006

\begin{tabular}{|c|c|c|c|c|c|c|c|c|}
\hline & \multicolumn{2}{|c|}{ PIBPM } & \multicolumn{2}{|c|}{ POBLACIÓN } & \multicolumn{2}{|c|}{ EMPLEO } & \multicolumn{2}{|c|}{ PIBpC } \\
\hline & $\begin{array}{l}\text { Crec. } \\
\text { medio }\end{array}$ & $\begin{array}{l}\text { Variación } \\
\text { del peso }\end{array}$ & $\begin{array}{l}\text { Crec. } \\
\text { medio }\end{array}$ & $\begin{array}{l}\text { Variación } \\
\text { del peso }\end{array}$ & $\begin{array}{l}\text { Crec. } \\
\text { medio }\end{array}$ & $\begin{array}{l}\text { Variación } \\
\text { del peso }\end{array}$ & $\begin{array}{l}\text { Nivel (miles } \\
€ / 2000)\end{array}$ & $\begin{array}{l}\text { Índice } \\
\text { (respecto a } \\
\text { España) }\end{array}$ \\
\hline ANDALUCÍA & 6,2 & $-0,7$ & 0,9 & 0,7 & 3,1 & 1,4 & 15.471 & 70,4 \\
\hline ARAGÓN & 5,6 & $-0,3$ & 0,3 & $-0,3$ & 1,9 & $-0,4$ & 21.099 & 96,0 \\
\hline ASTURIAS & 6,2 & $-0,1$ & $-0,2$ & $-0,6$ & 0,6 & $-0,9$ & 17.634 & 80,3 \\
\hline BALEARS (ILLES) & 7,0 & 0,1 & 2,1 & 0,5 & 6,7 & 0,9 & 21.678 & 98,7 \\
\hline CANARIAS & 5,6 & $-0,4$ & 1,9 & 0,9 & 2,9 & 0,1 & 17.450 & 79,4 \\
\hline CANTABRIA & 5,4 & $-0,2$ & 0,4 & $-0,1$ & 1,8 & $-0,2$ & 19.349 & 88,1 \\
\hline CASTILLA Y LEÓN & 4,9 & $-1,0$ & $-0,1$ & $-1,3$ & 1,2 & $-1,5$ & 18.587 & 84,6 \\
\hline CASTILLA - LA MANCHA & 4,7 & $-0,7$ & 0,6 & $-0,1$ & 2,5 & $-0,1$ & 15.281 & 69,6 \\
\hline CATALUÑa & 5,5 & $-2,2$ & 0,8 & 0,2 & 3,0 & 0,6 & 22.957 & 104,5 \\
\hline COMUNIDAD VALENCIANA & 5,1 & $-1,5$ & 1,3 & 1,1 & 3,1 & 0,5 & 17.758 & 80,8 \\
\hline EXTREMADURA & 6,7 & 0,0 & 0,0 & $-0,5$ & 1,6 & $-0,4$ & 13.289 & 60,5 \\
\hline GALICIA & 4,0 & $-1,6$ & $-0,1$ & $-1,3$ & 0,5 & $-2,6$ & 16.206 & 73,8 \\
\hline MADRID & 7,1 & 0,6 & 1,2 & 1,1 & 4,5 & 3,3 & 25.781 & 117,3 \\
\hline MURCIA (REGIÓN DE) & 4,8 & $-0,5$ & 1,7 & 0,5 & 3,9 & 0,4 & 16.360 & 74,5 \\
\hline NAVARRA & 5,7 & $-0,2$ & 0,7 & 0,0 & 2,7 & 0,0 & 24.510 & 111,6 \\
\hline PAÍS VASCO & 4,0 & $-1,9$ & 0,0 & $-0,9$ & 1,6 & $-1,0$ & 25.304 & 115,2 \\
\hline RIOJA & 3,0 & $-0,4$ & 0,8 & 0,0 & 2,6 & 0,0 & 21.072 & 95,9 \\
\hline TOTAL ESPAÑA & 6,7 & & 0,7 & & 2,7 & & 21.971 & 100,0 \\
\hline
\end{tabular}

FUENTE BD.MORES 2000 (2009).

NOTA PIBPM $=$ PIB A PRECIOS DE MERCADO; PIBPC = PIB PER CÁPITA.

El principal objetivo de esta sección es profundizar en las relaciones entre el crecimiento y la especialización de las regiones, prestando siempre particular atención a las actividades de servicios. Al respecto, puede anticiparse ya que la principal conclusión a la que se llega es el papel fundamental que desempeñaron los servicios en el crecimiento regional en España entre 1980 y 2006, sobre todo en términos de empleo.

El estudio de la contribución sectorial al crecimiento se realiza mediante el análisis de estandarización shift-share. Los resultados obtenidos utilizando esta metodología para el caso del sector servicios aparecen detallados en el Cuadro A.1 del Anexo. Se ha constatado que no existen diferencias notables entre el análisis del 
crecimiento en términos de empleo y el relativo a la producción, aunque sí que aparecen ciertas discrepancias que merecerán algunos comentarios.

La Figura 1 muestra los efectos de la estructura productiva (EP) y de las ventajas de localización regional (ED, efecto diferencial) sobre el crecimiento del empleo y el VAB (Valor Agregado Bruto) de las regiones durante el período estudiado. Destaca, al respecto, que solo el País Vasco, Andalucía, Cataluña, la Comunidad Valenciana, Baleares, Canarias y Madrid presentan valores positivos en términos de empleo durante dicho período. En términos de VAB, el número de regiones es mucho más reducido (Madrid, Andalucía, Baleares y Canarias). Dos regiones sobresalen de forma especial en el conjunto: el País Vasco y Cataluña, que en términos de empleo muestran un valor positivo del efecto estructura, lo cual no ocurre al utilizar datos del valor de la producción, donde el efecto es negativo y considerablemente bajo. Por otra parte, las regiones que muestran efectos estructura más negativos en términos de empleo son Galicia y Castilla y León.

FIGURA 1 | Efectos de la especialización productiva y las ventajas de localización en el crecimiento regional, 1980-2006

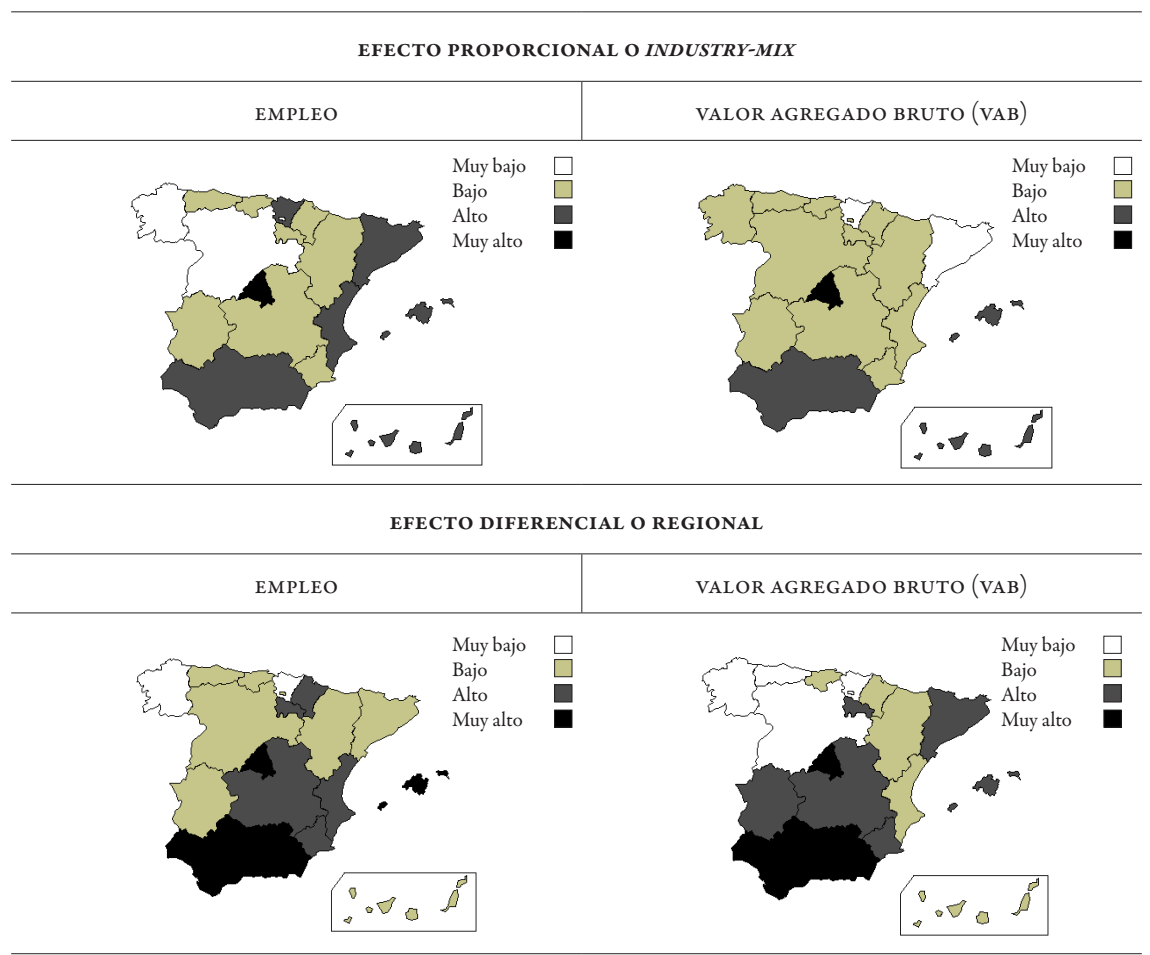

FUENTE ELABORACIÓN PROPIA.

El efecto diferencial o regional muestra valores y patrones de comportamiento muy diferentes. En primer lugar, el dinamismo de regiones como Castilla-La Man- 
cha, Murcia, Rioja, Baleares y, especialmente, Madrid y Andalucía, se refleja tanto en términos de empleo como de valor añadido. Por otra parte, algunas regiones como Navarra y la Comunidad Valenciana, Cataluña y Extremadura, únicamente reflejan dinamismo en términos de empleo o de valor añadido, respectivamente. Por el contrario, las regiones con una clara desventaja de localización (efecto diferencial negativo) son las de la llamada Cornisa Cantábrica (Galicia, Asturias, Cantabria y el País Vasco), junto con Castilla y León, Aragón y Canarias.

Atendiendo a la tipología regional que se especificó en el Cuadro 1, puede afirmarse que, en términos generales, la clasificación resultante no muestra variaciones notables. ${ }^{12}$ Las regiones más dinámicas del período fueron Madrid y Andalucía. Esto se debe al valor positivo del efecto total neto y al dinamismo que ambas regiones experimentaron, tanto desde el punto de vista de su especialización productiva como desde otros aspectos propios (la capitalidad, en el caso de Madrid, por ejemplo). En el extremo opuesto, dos regiones de la Cornisa Cantábrica (Galicia y Cantabria), junto con Aragón y Castilla y León se han caracterizado por tener una estructura productiva especializada en actividades poco dinámicas y efectos de localización negativos. Especial atención merece el caso de Asturias, ya que mientras en términos de empleo esta comunidad se encuadraría en el grupo de las regiones en declive, en términos de producción se ha comportado algo más dinámicamente.

Por su parte, Murcia presenta un efecto neto positivo gracias a los efectos de localización, ya que su estructura productiva está basada en actividades poco dinámicas. Lo contrario sucede en Canarias, cuyo efecto positivo se asienta únicamente en la positiva contribución de su componente estructural. Finalmente, comunidades como Navarra, Cataluña, La Rioja y Castilla-La Mancha han tenido un efecto neto negativo, a pesar de ciertos efectos de localización, debido a la composición de su estructura productiva. El País Vasco, a pesar de que su estructura productiva está asentada en actividades dinámicas, presenta ciertas desventajas de localización que han provocado un efecto neto total negativo.

Con objeto de profundizar en el papel que han desempeñado los servicios en las tendencias generales observadas, se ha llevado a cabo un análisis shift-share referido exclusivamente a dicho sector. El objetivo es comprobar cuáles han sido los efectos del cambio estructural dentro del propio sector servicios y los efectos de localización que se derivan en relación con el crecimiento en las diferentes regiones. Los resultados detallados se muestran en el Cuadro A.2 del Anexo y nos permiten subrayar algunos hechos.

En primer lugar, en el caso del efecto estructura o proporcional hay que destacar que, durante el período estudiado, solo Madrid, el País Vasco, Cataluña, Baleares y Cantabria presentan valores positivos en términos de empleo en servicios. En función del VAB, el número de regiones con efecto positivo es también reducido: Madrid, el País Vasco, Murcia, la Comunidad Valenciana y Cataluña. En el extremo opuesto, las regiones con efectos estructura más negativos fueron Andalucía y

No obstante, existen ciertos cambios significativos que necesitarían de un análisis caso por caso más detallado, tarea que no vamos a abordar en este trabajo, por cuestiones de oportunidad. 
Castilla y León. En segundo lugar, y por lo que se refiere al efecto diferencial o de localización, el dinamismo de regiones como Madrid, Baleares, Castilla-La Mancha, Murcia o La Rioja se refleja tanto en términos de empleo como de valor añadido. Sin embargo, algunas regiones — como la Comunidad Valenciana, o Cataluña y Andalucía - solo muestran dinamismo en términos de empleo o de valor añadido, respectivamente. Por contra, las regiones con un claro efecto diferencial negativo siguen localizándose en la Cornisa Cantábrica (País Vasco, Galicia, Asturias y Cantabria), junto con Castilla y León, Canarias y Aragón.

Por último, atendiendo a las tipologías de clasificación regional subyacentes en el análisis shift-share y con referencia al sector servicios, hay que subrayar que la única región dinámica en el período ha sido Madrid, tanto en empleo como en VAB. Otras dos CC.AA., Comunidad Valenciana y Baleares, también se comportaron dinámicamente en términos de empleo, aunque no así en valor añadido. En el extremo opuesto, dos de las regiones cantábricas (Galicia y Asturias), junto con Castilla y León, Navarra, Aragón, Extremadura y Canarias se caracterizan por tener tanto una estructura terciaria especializada en servicios poco dinámicos (Comercio y hostelería), como desventajas en términos de localización. El País Vasco, a pesar de su estructura terciaria asentada en actividades dinámicas (finanzas, servicios a empresas...), presenta ciertas desventajas de localización que han provocado que el efecto neto total sea moderadamente negativo.

Como puede observarse, muchos de los comportamientos o tipologías dentro del sector servicios son, en buena medida, una mera traslación de lo que se obtenía al estudiar los sectores básicos en su conjunto. Esto puede atribuirse, esencialmente, al elevado peso - tanto cuantitativo como estratégico- que supone el sector servicios en las economías regionales españolas.

\section{Una nota en torno al posible impacto de la crisis iniciada a partir de mediados de 2008}

Tal como se ha indicado anteriormente, la base utilizada en este trabajo no ha incorporado todavía los datos correspondientes a los ejercicios posteriores a 2006, que sin duda estarán marcados por la profunda crisis que ha afectado y que todavía afecta a la economía española. Téngase en cuenta que de un crecimiento del PIB español del 3,6\% en 2007, en valores reales, se pasó a solo un 0,9\% en 2008 y a una tasa negativa del $-3,7 \%$ en 2009 , que todavía siguió siendo ligeramente negativa $(-0,1 \%)$ en 2010. Las estimaciones para 2011 sitúan el crecimiento en valores positivos, aunque con una tasa de tan solo $0,7 \%$. El resultado más penoso de este proceso ha sido el fuerte incremento del desempleo, que ha pasado de alrededor de $8 \%$ de la población activa en 2007 a situarse algo por encima del 20\% en el primer trimestre de 2011. Esto representa un incremento de más de dos millones de desocupados más que los que había a principios de 2008.

Las causas que explican esta negativa evolución de la economía española derivan, aunque solo en parte, de los efectos de la crisis financiera internacional. Por 
supuesto que esta ha acabado repercutiendo en España, sobre todo como consecuencia del elevado endeudamiento de las entidades financieras con el exterior y de la creciente necesidad de financiar el déficit público, puesto que los estabilizadores automáticos, junto con la expansión del gasto discrecional no productivo que se llevó a cabo al inicio de la recesión, han provocado un intenso deterioro de las cuentas públicas. Pero la crisis y la fuerte recesión española han tenido un claro protagonista interno: el brusco fin de la burbuja inmobiliaria que se había alimentado desde 2002-2003 y el profundo desplome de la construcción, que ha arrastrado a su vez a las numerosas ramas productivas (industriales y de servicios) directa e indirectamente vinculadas a dicho sector. A todo ello se han sumado los efectos del desempleo y de la caída de actividad en el consumo privado y los reajustes a los que se ha visto sometido todo el sector financiero español. La grave crisis de la deuda soberana en la Eurozona y las dudas sobre la capacidad de España para llevar a cabo los inevitables ajustes y reformas han situado recientemente a la economía española en el punto de mira de los mercados, lo que se ha traducido más recientemente en un incremento de los costes y una mayor dificultad de acceso a la financiación. ${ }^{13}$

Aunque se dispone de algunos datos básicos sobre la evolución de las economías regionales en 2007, 2008, 2009 e incluso de un avance de $2010,{ }^{14}$ con ellos no es posible ampliar los análisis de este trabajo para el período 1980-2006. Ello sobre todo porque la desagregación por grandes sectores y el detalle para las distintas ramas de servicios no están disponibles.

Cabe, con todo, señalar algunos cambios importantes que se han producido y que han afectado de forma desigual a las distintas CC.AA. españolas Así, las estimaciones referidas al ejercicio 2009, que — como se ha indicado- registró una tasa negativa de $-3,7 \%$ en el PIB nacional, ofrece diferencias importantes a escala regional. En Extremadura, la región menos afectada, la tasa negativa de variación de su PIB en dicho ejercicio fue del $-2,05 \%$, mientras que Cataluña $(-4,36 \%)$ y Aragón (-4,66\%) ocupan las peores posiciones. La caída de la construcción ha afectado intensamente a las CC.AA. de la costa mediterránea (Cataluña, Comunidad Valenciana, Murcia y Andalucía), pero también a algunas metrópolis y zonas del interior (Madrid, Castilla-La Mancha) y a las islas Canarias y Baleares. El sector manufacturero solo creció un $0,7 \%$ en 2007 (cuando la economía en su conjunto lo hizo todavía en un 3,6\%), pasando a tasas negativas del $-2,7 \%$ y del $-13,6 \%$ en 2008 y 2009 , respectivamente. La construcción aún crecía en 2007 (2,5\%), pero registra tasas negativas en todos los ejercicios siguientes: - 1,6 en 2008; -6,2\% en 2009; -6,3\% en 2010.

El impacto negativo de la crisis en los servicios ha sido menos profundo que en la industria y la construcción. En el conjunto español, los servicios de mercado solamente registraron una tasa negativa de crecimiento en 2009 (-1,9\%), mientras que

13 No tendría sentido extenderse aquí en más detalles sobre la evolución de la economía, las dificultades que España tiene para recuperar una senda de crecimiento aceptable y el tipo de reformas y ajustes que todavía es preciso llevar a cabo, además de los que ya se han implementado en los últimos meses.

14 Nos referimos a los datos oficiales publicados por el INE hasta 2009 y a los que ha anticipado la Fundación de las Cajas de Ahorros (Funcas), de carácter no oficial, para los cuatro años citados. 
en 2008 el sector creció un 1,7\% y en 2010 lo hizo en $0,8 \%$, cuando la tasa media del PIB español fue negativa $(-0,1 \%)$. Esta evolución ha quedado en parte compensada por el comportamiento de los SNDV, que registraron tasas siempre positivas en 2008 (4,7\%), 2009 (2,1\%) y 2010 (0,8\%), aunque decrecientes debido a los ajustes que está llevando a cabo el sector público, tanto estatal como regional. Desde el punto de vista del empleo, los servicios todavía fueron capaces de generarlo en 2008, y en 2009 la caída del empleo terciario fue mucho menos acusada (2,5\%) que la del total de la economía (6,8\%). Por último, en 2010, el sector ha sido capaz de incrementar el empleo neto respecto a un año antes. En consecuencia, en los tres años de la crisis, los servicios solo redujeron el empleo en 1,6\%, frente al 10\% de la economía en su conjunto, el 42,8\% de la construcción y el 19,4\% de la industria.

Desde la óptica regional, la evolución del sector servicios ha mostrado comportamientos diferenciados, aunque no muy dispares. Así, el retroceso del turismo en 2008 y 2009 afectó principalmente a los dos archipiélagos (Canarias y Baleares) y a las regiones costeras mediterráneas, recuperándose ligeramente en 2010. Las ramas de servicios más vinculadas a la industria y al gasto de los consumidores anticipan datos regionales algo peores que la media del país, y el comportamiento de los SNDV ha favorecido a las regiones que tienen un mayor grado de especialización en este subsector. Pero, como se ha indicado, la crisis ha tenido mucha menos incidencia en el sector servicios que en el resto de las ramas de actividad. Ello permite anticipar que sus efectos seguramente no han incidido en buena parte de las conclusiones obtenidas anteriormente al estudiar el largo período 1980-2006. En todo caso, solo cuando se disponga de datos fiables por sectores y para los distintos subsectores de servicios será posible contrastarlo adecuadamente. Lo que parece indudable es que la crisis ha acelerado - aunque no definitivamente - la terciarización de la economía española y la de muchas de sus regiones. Al cerrar 2010, los servicios mantenían una elevada cifra de ocupación (13.402,2 mil personas) a escala nacional, equivalente al 72,6\% del empleo total español, cuando en 2007 representaba un $66,4 \% .^{15}$

\section{Notas finales}

Como se expuso en la introducción, el principal objetivo de este trabajo ha sido presentar, de forma muy sintética, las grandes tendencias de la especialización productiva regional en España a partir de 1980, prestando especial atención a las relacionadas con el sector servicios en su conjunto y sus principales subsectores. Como se recordará, las dos hipótesis de partida eran: i) que la posible existencia en España de una notable inercia en la especialización determina una baja convergencia de las estructuras productivas regionales; y ii) que la especialización productiva influye en el crecimiento económico de las regiones y que los servicios han desempeñado un indudable papel en este sentido. Este trabajo se ha orientado a

15 Datos tomados de las fuentes oficiales del INE. Varios documentos e institutos coinciden en sus análisis con las opiniones manifestadas sobre la crisis y los servicios. Véase Informe del Banco de España (2010); informes del Instituto Flores de Lemus y de Funcas. 
contrastar ambas hipótesis, ofreciendo algún tipo de respuesta a las preguntas que se formularon en la introducción.

La terciarización de la economía española ha sido realmente intensa en las últimas décadas. Esto se ha trasladado a todas las regiones, aunque no de forma homogénea. De hecho, en el largo período estudiado se aprecian diversos patrones de especialización en las CC.AA. españolas. Así, las regiones más especializadas en servicios no suelen mostrar especializaciones significativas en sectores como la agricultura o las manufacturas, mientras que sí mostraron una relación más significativa con la especialización en la construcción, especialmente en las regiones más turísticas.

El análisis más desagregado del sector servicios ha permitido añadir algunos matices de indudable interés. En concreto, algunas ideas que cabe extraer del trabajo realizado confirman las hipótesis que figuraban en el punto de partida. Así, el estudio de la evolución de las estructuras productivas regionales ha mostrado la existencia de una notable inercia temporal en los cambios y en la especialización de las CC.AA. Dicha inercia, que se aprecia en cuanto al conjunto de los principales sectores productivos, se manifiesta también en la composición interna de los servicios. ${ }^{16}$ En este sentido, el ranking de especialización regional en servicios es muy estable en todo el período analizado, lo cual no impide que se registren algunos cambios en la posición de varias CC.AA., ni que también los haya en la composición interna del sector servicios, si bien no pueden calificarse como muy importantes.

El proceso de aproximación de las estructuras productivas de las regiones ha sido, en cuanto al conjunto de los sectores básicos, bastante escasa. Por el contrario, cabe afirmar que sí hay un grado de convergencia más marcado en los servicios y en su composición interna, aunque persiste la heterogeneidad que ya existía al inicio del período estudiado.

El análisis de las relaciones entre crecimiento y especialización regional permite afirmar que los efectos diferenciales en el crecimiento muestran patrones de comportamiento claramente diferentes por sectores y regiones. Parece innegable, ante todo, que los servicios han tenido una fuerte influencia en la expansión de las CC.AA. Los efectos vinculados a la estructura productiva y las ventajas de localización regional ofrecen resultados de signo claramente positivo en varios casos. El análisis de descomposición permite concluir, en concreto, que el crecimiento regional es un fenómeno caracterizado por la heterogeneidad de comportamientos. Pese a esto, es posible establecer ciertos patrones comunes para distinguir entre las regiones con buenos resultados, o dinámicas, y aquellas otras que podrían considerarse en declive. El análisis shift-share ha permitido establecer qué factores de localización o de especialización han impulsado el crecimiento regional en España desde principios de los ochenta.

A expensas de los matices que ofrecen los datos obtenidos en la sección "Especialización productiva y crecimiento de los servicios”, el País Vasco, Andalucía, Cataluña, la Comunidad Valenciana, Madrid y las dos regiones insulares (Baleares y Cana-

Conviene recordar que la desagregación por ramas de servicios que ofrece la base estadística utilizada es muy limitada. Seguramente una desagregación mucho más detallada permitiría matizar e incluso cuestionarse esta afirmación general, más si cabe en un sector tan heterogéneo como el terciario, tanto a nivel nacional (Cuadrado \& González, 2009) como regional (Cuadrado \& Maroto, 2010a). 
rias) muestran valores positivos en términos de empleo. Sin embargo, el número de regiones con valores positivos se reduce cuando se toma como referencia el VAB, ya que solo destacan Madrid, Andalucía, Canarias y Baleares. Por otra parte, el efecto diferencial muestra patrones de comportamiento claramente distintos. Las regiones con evidentes desventajas de localización en el período estudiado - efecto diferencial negativo - son las de la Cornisa Cantábrica (Galicia, Asturias, Cantabria y el País Vasco), junto con Castilla y León, Aragón e incluso Canarias.

Los cálculos realizados permitirían incorporar otros resultados de interés, que aparecen reseñados anteriormente. Pero, en cualquier caso, es necesario recordar que, si bien no cabe cuestionarlos globalmente, la insuficiente desagregación de la base de datos utilizada influye en algunos resultados. Lo que sí sugiere este trabajo es la conveniencia de profundizar en el comportamiento específico de algunas regiones, tarea que queda abierta a otras investigaciones de carácter monográfico.

Finalmente, la crisis que está viviendo la economía española desde la segunda mitad de 2008 - aunque en la construcción se inició algo antes- ha supuesto un profundo giro que, sin duda, está teniendo su reflejo a escala regional. La no disponibilidad de datos a este nivel impide extender los análisis realizados en este trabajo con el fin de estudiar los efectos de la crisis. Sin embargo, conviene señalar que el sector servicios ha tenido un comportamiento mucho mejor que el resto de la economía, lo que sugiere que las tendencias de largo alcance que este trabajo aporta en cuanto a la relación entre los servicios y las economías regionales seguramente no van a verse invalidadas. Pero lo que sí será obligado es someterlo a la necesaria contrastación.

\section{Referencias bibliográficas}

Adams, J. \& Pigliaru, F. (Eds.) (1999). Economic growth and change. National and regional patterns of convergence and divergence. Cheltenham, UK/Northampton, MA: Edward Elgar.

Amiti, M. (1999). Specialization patterns in Europe. Review of World Economics, 135(4), 573-593.

Armstrong, H. \& Taylor, J. (2000). Regional Economics and Policy (3a ed.). Oxford: Blackwell.

Brockett, P. L., Golany, B. \& Li, S. (1999). Analysis of intertemporal efficiency trends using rank statistics with an application evaluating the macro economic performance of OECD nations. Journal of Productivity Analysis, 11(2), 169-82

Brockett, P. L. \& Kemperman, J. (1980). Statistical recognition of trends in health monitoring systems. Methods of Information in Medicine, 19(2), 106-112.

Camagni, R. \& Cappellin, R. (1985). La productivité sectorielle et la politique régionale. Bruselas: Comisión Europea.

Capello, R. (2004). Economia Regionale. Bolonia: Il Mulino.

Combes, P. \& Overman, H. (2003). The spatial distribution of economic activities in the European Union. En V. Henderson \& J. Thisse (Eds.), Handbook of Urban and Regional Economics, vol. 4. Ámsterdam: North-Holland.

Cuadrado-Roura, J. R. (2008). Los servicios, sector clave en la expansión e internacionalización de la economía. En J. Velarde \& J. M. Serrano (Eds.), Economía. España siglo XXI (cap. 9, pp. 327-377). Madrid: Biblioteca Nueva e Instituto de España. 
Cuadrado-Roura, J. R., García-Greciano, B. \& Raymond, J. L. (1999). Regional convergence in productivity and productive structure: The Spanish case. International Regional Science Review, 22(1), 35-53.

Cuadrado-Roura, J. R., Garrido, R. \& Mancha, T. (2002). Productivity patterns in Europe: an alternative approach. Annals in Regional Science, 34(3), 333-56.

Cuadrado-Roura, J. R. \& González, M. (2009). Los servicios en el proceso de crecimiento de España. Papeles de Economía Española, 120, 2-27.

Cuadrado-Roura, J. R. \& Maroto, A. (2008). Regional convergence in productivity and productive structure in Mediterranean countries. Comunicación presentada al $48^{\text {th }}$ ERSA Congress, Liverpool.

Cuadrado-Roura, J. R. \& Maroto, A. (2010a). Servicios y regiones en España. Estudios de la Fundación, n. ${ }^{\circ} 29$. Madrid: Fundación de las Cajas de Ahorros (Funcas).

Cuadrado-Roura, J. R. \& Maroto, A. (2010b). Regional productivity convergence and changes in the productive structure. En J. R. Cuadrado (Ed.), Regional policy, economic growth and convergence. Lessons from the Spanish case (pp. 149-170). Heidelberg, Dordrecht, Londres y Nueva York: Springer.

Cuervo, L. M. (2004). Estudios de convergencia y divergencia regional en América Latina. Balance y perspectivas. Investigaciones Regionales, n. 5 (otoño), 29-65.

Chávez, F. (1995). Los servicios en México: crecimiento, empleo, rentabilidad. México: Universidad Autónoma Metropolitana, Unidad Azcapotzalco.

De Bustos, A., Cutanda, A., Díaz, A., Escribá, F. J., Murgui, M. J. \& Sanz, M. J. (2008). La BD.MORES en base 2000. Nuevas estimaciones y variables. Documento de Trabajo 2008-02. Madrid: Dirección General de Presupuestos, Ministerio de Economía y Hacienda.

Domenech, R. (2008). La evolución de la productividad en España y el capital humano. Documento de Trabajo 141/2008. Madrid: Laboratorio de Alternativas, Fundación de Alternativas.

Eatwell, J., Milgate, M. \& Newman, P. (1989). Economic Development. Nueva York y Londres: New Palgrave, WW-Norton.

Eckey, H. \& Türk, M (2007). Convergence of EU regions. A literature report. Investigaciones Regionales, n. ${ }^{\circ} 10,5-32$.

Esteban, J. (2000). Regional convergence in Europe and the industry mix. A shift.share analysis. Regional Science and Urban Economics, 30(3), 353-364.

Estrada, A., Jimeno, J. F. \& Malo de Molina, J. L. (2009). La economía española en la UEM: los diez primeros años. Documentos ocasionales, n. ${ }^{0}$ 0901. Madrid: Banco de España.

Ezcurra, R., Pascual, P. \& Rapún, M. 2006. The dynamics of industrial concentration in the regions of the European Union. Growth and Change, 37(2), 200-229.

Fagerberg, J. (2000). Technological progress, structural change and productivity growth. A comparative study. Structural Change and Economic Dynamics, 11(4), 393-411.

Garrido, R. (2002). Cambio estructural y desarrollo regional en España. Madrid: Pirámide.

Garza, G. (2006). La organización espacial del sector servicios en México. México DF: El Colegio de México.

Hallet, M. (2000). Regional specialization and concentration in the European Union. Economic Papers [European Commission], n. ${ }^{\circ} 141,1-29$. 
Kuznets, S. (1974). Crecimiento económico y estructura económica (2a ed.). Barcelona: Ariel.

Maroto, A. \& Cuadrado, J. R. (2006). La productividad de la economía española. Madrid: Instituto de Estudios Económicos, Colección Estudios.

Maroto, A. \& Cuadrado, J. R. (2007). Productivity and tertiarization in industrialized countries. A comparative analysis. Efficiency Working Series. Oviedo: Universidad de Oviedo.

Maroto, A. \& Cuadrado, J. R. (2008). Evolución de la productividad en España. Un análisis sectorial, 1980-2006. Economía Industrial, n. ${ }^{\circ} 367,15-34$.

Maroto, A. \& Cuadrado, J. R. (2009). Is growth of services an obstacle to productivity growth? A comparative analysis. Structural Change and Economic Dynamics; 20(4): 254-65

Organisation for Economic Co-operation and Development (OECD). (2009). How regions grow. Trends and analysis. París: Autor.

Paci, R. \& Pigliaru, F. (1999). Growth and sectoral dynamics in the Italian regions. En J. Adams \& F. Pigliaru, (Eds.), Economic growth and change. National and regional patterns of convergence and divergence (pp. 291-322). Cheltenham, UK/Northampton, MA: Edward Elgar.

Prebisch, R. (1950). The economic development of Latin America and its principal problems. Nueva York: Organización de las Naciones Unidas.

Peneder, M. (2002). Industrial structure and aggregate growth. WIFO [Austrian Institute of Economic Research] Working Papers, 182, Viena.

Peneder, M. (2003). Industrial structure and aggregate growth. Structural Change and Economic Dynamics, 14(4), 427-448.

Raymond, J. L. (1990). Estructura productiva y grado de diversificación sectorial de las Comunidades Autónomas. Papeles de Economía Española, n. ${ }^{\circ} 45,23-24$.

Segura, J. (Coord.) (2006). La productividad en la economía española. Madrid: Fundación Ramón Areces.

Suárez-Villa, L \& Cuadrado-Roura, J. R. (1996). Thirty years of Spanish regional change. Interregional dynamics and sectorial transformation. En K. Haynes \& K. Button (Eds.), Regional Dynamics (vol. 1, pp. 217-252). Cheltenham, UK y Brookfield, VT: Edward Elgar.

Thirwall, A. P. (1999). Growth and Development (6a ed.). Hampshire y Londres: MacMillan.

Temple, M. (1994). Regional Economics. Nueva York: St. Martin’s Press.

Timmer, M. \& Szirmai, A. (2000). Productivity growth in Asian manufacturing: the structural bonus hypothesis examined. Structural Change and Economic Dynamics, 11(4), 371-392.

Todaro, M. P. (1982). Economía para un mundo en desarrollo. México, DF: Fondo de Cultura Económica.

Van Ark, B. (1995). Sectoral growth and structural change in post-war Europe. Research Memorandum GD-23. Groningen: The Groningen Growth and Development Centre (GGDC). 